Gut, 1981, 22, 213-216

\title{
Pentagastrin in diffuse oesophageal spasm
}

\author{
R M WEXLER, AND M D KAYE*
}

From the Department of Medicine, University of Vermont College of Medicine, Burlington, Vermont, USA

SUMMARY The effect of pentagastrin on oesophageal motility was studied in six subjects with idiopathic diffuse resophageal spasm (IDOS). Pentagastrin was administered by continuous intravenous infusion in doses of $1 \mu \mathrm{g} / \mathrm{kg} / \mathrm{h}, 5 \mu \mathrm{g} / \mathrm{kg} / \mathrm{h}$, and $10 \mu \mathrm{g} / \mathrm{kg} / \mathrm{h}$. Saline infusion was used as a control. No subject experienced pain during pentagastrin infusion. Two developed dysphagia and repetitive contractions with 'wet' swallows during the saline infusion and the lowest pentagastrin infusion. Contraction amplitude was increased only with 'dry' swallows during the $10 \mu \mathrm{g} / \mathrm{kg} / \mathrm{h}$ infusion period. Contraction duration was increased with both 'wet' and 'dry' swallows during the $1 \mu \mathrm{g} / \mathrm{kg} / \mathrm{h}$ infusion, and with 'dry' swallows during the $10 \mu \mathrm{g} / \mathrm{kg} / \mathrm{h}$ infusion. Propagation velocity was not altered by pentagastrin. We conclude that gastrin released physiologically by eating probably does not contribute to symptom production in IDOS. Moreover, it seems unlikely that pentagastrin, at least in these doses, can be exploited for diagnostic purposes.

Idiopathic diffuse oesophageal spasm (IDOS) is a disorder characterised by dysphagia, chest pain, or both. In typical cases, manometry reveals prolonged, powerful, repetitive, and simultaneous contractions in the lower two-thirds of the oesophageal body. Although oesophageal spasm can occur as a result of a variety of underlying disorders, ${ }^{1}$ the aetiology of the primary syndrome remains obscure. Eckhardt, Kruger, Holtermuller, and $\mathrm{Ewe}^{2}$ first noted that oesophageal spasm could be precipitated by pentagastrin in patients with IDOS. Hollis, Levine, and Castell ${ }^{3}$ had previously shown that similar doses of pentagastrin given to normal subjects increased the lower oesophageal sphincter pressure but did not affect motility in the oesophageal body. The observations of Eckhardt et al. ${ }^{2}$ were confirmed by others ${ }^{4}$ and led to the speculation that supersensitivity to endogenous gastrin might underlie the symptoms in this disorder. ${ }^{6}$ Further, it appeared that pentagastrin administration might prove useful as a provocative test to aid in diagnosis of the syndrome in patients whose radiographic and manometric abnormalities were not clearcut. However, other investigators have found no supersensitivity to gastrin ${ }^{7}$ or supersensitivity only when gastrin is administered in pharmacological doses. ${ }^{5}$

We set out to determine the effects of physiological

*Address for correspondence: Michael D Kaye, University of Vermont, College of Medicine, Given Building, C307, Burlington, Vermont 05405, USA

Received for publication 16 October 1980 and supra-physiological doses of pentagastrin in patients with IDOS. We included in our analysis the effect of pentagastrin on propagation velocity of oesophageal contractions, an effect not previously defined. Our experiment was designed to provide relatively stable serum pentagastrin levels, to control for placebo effect, and to avoid bias.

\section{Methods}

Six subjects with IDOS were studied. All had dysphagia, chest pain, or both. Prominent tertiary waves or a corkscrew appearance on barium swallow, and simultaneous or repetitive contractions of increased amplitude and duration on manometry were present in all subjects. Lower oesophageal sphincter relaxation was demonstrable, though not always complete, in all patients. When clinically indicated, oesophagoscopy and biopsy were used to exclude coexistent oesophagitis. There were three male and three female subjects, with a mean age of 50 years. All gave informed consent; and the experimental protocol was approved by the Committee on Human Experimentation at the University of Vermont.

Fasting subjects were studied with a triple lumen tube complex with three openings for pressure transmission at $5 \mathrm{~cm}$ intervals. The most distal opening was positioned $2 \mathrm{~cm}$ above the upper margin of the lower oesophageal sphincter. Each catheter was perfused continuously with water $(0.6 \mathrm{ml} / \mathrm{min})$ by a 
pneumohydraulic capillary infusion system (Arndorfer Medical Specialities Co.). Each catheter was connected to an external transducer (Bell and Howell, P327-1) whose output was recorded on a multichannel recorder (Gilson Macropolygraph). Respiration and swallowing were recorded by pneumograph and myograph respectively.

The study was divided into five $\mathbf{2 0}$ minute infusion periods as follows:

120 minute saline infusion.

2, 3, 420 minute infusions of pentagastrin in dosages of $1 \mu \mathrm{g} / \mathrm{kg} / \mathrm{h}, \quad 5 \mu \mathrm{g} / \mathrm{kg} / \mathrm{h}, \quad$ and $10 \mu \mathrm{g} / \mathrm{kg} / \mathrm{h}$. Saline was infused for five minutes between each pentagastrin infusion.

520 minute saline infusion.

Constant intravenous infusion was achieved by use of a Harvard infusion pump. The concentration of pentagastrin in the perfusate was adjusted to obtain the desired dosage.

Each 20 minute infusion period was further subdivided. The first five minutes were reserved for equilibration. Spontaneous motor activity was recorded during the next five minutes. During the final 10 minutes the responses to 10 'dry' and 10 'wet' ( $5 \mathrm{ml}$ of water at room temperature) swallows were recorded in six and four subjects respectively. Thirty seconds were allowed to elapse between swallows.

Tracings were analysed with respect to the following: contraction amplitude, contraction duration, propagation velocity, and frequency of spontaneous and repetitive contractions. Tracings were separated into their component portions, coded, and analysed by one of us who had not been present during the study, and who was therefore unaware of the particular infusion that corresponded to the tracing at hand.

\section{Results}

Pain did not occur during pentagastrin infusion. Two subjects had dysphagia and regurgitation with 'wet' swallows during both the first saline infusion and the $1 \mu \mathrm{g} / \mathrm{kg} / \mathrm{h}$ pentagastrin infusion. These symptoms were accompanied by numerous repetitive contractions and were sufficiently severe to preclude further study with 'wet' swallows in these individuals.

Values obtained during the first and second periods of saline infusion were similar. Hence, control data represent the average for these two periods.

The amplitude and duration of oesophageal contractions were compared with control values in several ways. The mean values from specific oesophageal positions $(2 \mathrm{~cm}, 7 \mathrm{~cm}$, and $12 \mathrm{~cm}$ above LOS), as well as the means derived by pooling values from all three positions, were compared with the appropriate controls. Comparisons of amplitude and durations were made also using means of the five highest alues, and means after exclusion of the two highest and the two lowest values.

Despite these manipulations, differences between saline and pentagastrin infusions were very small, and few were statistically significant (P 0.05). The results are summarised in the Table. For values obtained from pooled positions, contraction amplitude was significantly increased only with 'dry' swallows and only during the $10 \mu \mathrm{g} / \mathrm{kg} / \mathrm{h}$ infusion $(15.15 \mathrm{kPa} v s 13.75 \mathrm{kPa})$. Contraction duration was increased with both 'wet' and 'dry' swallows during the $1 \mu \mathrm{g} / \mathrm{kg} / \mathrm{h}$ infusion $(8.40 \mathrm{~s} v s 7.67 \mathrm{~s}$, 'dry'; and $9.05 \mathrm{~s} v \mathrm{~s} 7.66 \mathrm{~s}$, 'wet'). There was no increase in contraction duration with the $5 \mu \mathrm{g} / \mathrm{kg} / \mathrm{h}$ infusion. The duration after 'dry', but not 'wet', swallows was increased (8.49 s vs $7.67 \mathrm{~s})$ during the $10 \mu \mathrm{g} / \mathrm{kg} / \mathrm{h}$ infusion. Analysis of these same parameters by the additional methods described above did not reveal greater differences between the pentagastrin and control values.

As shown in the Table, propagation velocity was not affected by pentagastrin. Similarly, with the 'wet' swallow data from the two symptomatic subjects excluded, pentagastrin did not increase the frequency of spontaneous or repetitive contractions.

\section{Discussion}

In our study the effects of pentagastrin on oesophageal contraction amplitude and duration were

Table Effects of pentagastrin on amplitude, duration, and propagation time of oesophageal contraction

\begin{tabular}{|c|c|c|c|c|c|c|}
\hline \multirow[t]{2}{*}{$P G(\mu g / k g / h)$} & \multicolumn{2}{|c|}{ Amplitude $(k P a)$} & \multicolumn{2}{|c|}{ Duration (s) } & \multicolumn{2}{|c|}{ Propagation time $(\mathrm{sec} / 5 \mathrm{~cm})$} \\
\hline & 'Dry' & 'Wet' & 'Dry' & 'Wet' & 'Dry' & 'Wet' \\
\hline 0 (control) & $13 \cdot 75$ & $14 \cdot 46$ & $7 \cdot 67$ & $7 \cdot 66$ & $0 \cdot 27$ & 0.37 \\
\hline 1 & $15 \cdot 33$ & $15 \cdot 22$ & $8 \cdot 40^{*}$ & $9 \cdot 05 *$ & $0 \cdot 37$ & $0 \cdot 29$ \\
\hline 5 & $14 \cdot 54$ & $15 \cdot 35$ & $8 \cdot 13$ & $7 \cdot 94$ & $0 \cdot 22$ & $0 \cdot 26$ \\
\hline
\end{tabular}

Significantly $(\mathrm{P}<0.05)$ difterent from control, Student's paired $t$ test. 
very small; and pentagastrin had no demonstrable effect on propagation velocity. We used doses of pentagastrin that were in both the physiological and pharmacological range as defined by acid secretory studies. ${ }^{8}$ There was no direct dose-response relationship. From these results it is unlikely that endogenous gastrin released during eating contributes significantly to symptom production in most patients with this disorder. In addition, it seems unlikely that pentagastrin will be a useful provocative agent to aid in the diagnosis of the disorder.

Our results are in apparent conflict with some of those published previously. There are several possible explanations, the most important of which, we believe, is the difference between our experimental design and that of others. Whereas in most previous studies pentagastrin has been administered as an intravenous bolus or by subcutaneous injection, we chose to give it by continuous intravenous infusion in order to achieve more controlled and sustained blood levels. When given as an intravenous bolus, pentagastrin has only a very transient effect. It has been shown in dogs that gastrin fragments of less than nine amino acid residues are more than $90 \%$ inactivated by hepatic transit. ${ }^{9}$ After subcutaneous injection, blood levels of pentagastrin are variable and unpredictable. Moreover, lower oesophageal sphincter pressure in normal subjects reaches a peak as early as five minutes after subcutaneous injection, and then quickly returns to baseline. ${ }^{3}$ In addition, we used saline infusion as a control. This provides a placebo injection, which is pertinent, as it has long been recognised that emotional stress can induce spasm in patients with oesophageal motor disorders. ${ }^{10-12}$. It helps also to control for aspects of the experimental protocol, other than pentagastrin, which might induce spasm in patients with IDOSfor example, the oesophageal catheter itself. By having two control saline infusions, one before and one after the pentagastrin infusions, we attempted to determine if there were any time-related variables, such as fatigue, which might bias our results. While the similarity of results from these two control periods argues against any such variable, it should be stressed that two subjects became symptomatic after the 'wet' swallows in the early stages of the experimental protocol. The vigorous repetitive contractions which coincided with their symptoms of dysphagia and regurgitation were almost certainly due to water retained in the oesophagus because of impaired emptying in the supine position. We have observed a similar phenomenon in other patients with oesophageal spasm not included in this particular study. Clearly, if such patients are included in protocols which examine the effects of an agent such as pentagastrin after control observations, changes in motility may be attributed wrongly to that agent. Finally, in an attempt to eliminate observer bias, all tracings were read in a blinded fashion. This is particularly important, as the characteristics of oesophageal contractions in individual patients with IDOS are highly variable.

Other factors, unrelated to methodology, may be relevant to the disparity between our results and those of others. Firstly, it is possible that there are subgroups of patients with IDOS that vary in their sensitivity to gastrin and its analogues, and that our population of subjects was skewed in this regard. Secondly, our number of subjects may have been too small to disclose a more consistent pentagastrin effect. Thirdly, it is possible that larger doses of pentagastrin might have produced more obvious effects. We feel that this is unlikely, since there was no direct dose-response relationship over the range $(1-10 \mu \mathrm{g} / \mathrm{kg} / \mathrm{h})$ which we studied.

There is an important subgroup of patients whose symptoms are identical with those of patients with IDOS, but whose radiographs and manometric records are either normal or not sufficiently abnormal to justify a confident diagnosis of oesophageal spasm. There is clearly a need for some provocative diagnostic agent in such patients, and it was our hope that pentagastrin might fill this role. As the effects of pentagastrin in our patients with typical IDOS were so inconspicuous, we must conclude, with regret ,and in accord with Orlando and Bozymski, ${ }^{5}$ that it will not.

\section{References}

${ }^{1}$ Bennett JR, Hendrix TR. Diffuse oesophageal spasm: a disorder with more than one cause. Gastroenterology 1970; 59: 273-9.

${ }^{2}$ Eckhardt VF, Kruger J, Holtermuller $\mathbf{K H}$, Ewe $\mathbf{K}$. Alteration of oesophageal peristalsis by pentagastrin in patients with diffuse oesophageal spasm. Scand $J$ Gastroenterol 1975; 10: 475-9.

${ }^{3}$ Hollis JB, Levine SM, Castell DO. Differential sensitivity of the human oesophagus to pentagastrin. $A m J$ Physiol 1972; 222: 870-4.

${ }^{4}$ Lane WH, Ippoliti AF, McCallum RW. Effect of gastrin heptadecapeptide (G17) on oesophageal contractions in patients with diffuse oesophageal spasm. Gut 1979; 20: 756-9.

${ }^{5}$ Orlando RC, Bozymski EM. The effects of pentagastrin in achalasia and diffuse oesophageal spasm. Gastroenterology 1979; 77: 472-7.

${ }^{6}$ Cohen S. Symptomatic diffuse oesophageal spasm and its relation to gastrin supersensitivity. Ann Intern Med 1975; 82: 714-5.

${ }^{7}$ Morris SJ, Perez C, Rogers AI. Sensitivity of oesophageal peristalsis to pentagastrin (PG) in patients with syptomatic diffuse oesophageal spasm (abstract). Gastroenterology 1978; 74: 1137. 
${ }^{8}$ Multicentre Pilot Study. Pentagastrin as a stimulant of maximal gastric acid response in man. Lancet 1967; 1 : 291-5.

'Strunz UT, Thompson MR, Elashoff J, Grossman MI. Hepatic inactivation of gastrins of various chain lengths in dogs. Gastroenterology 1978; 74: 550-3.

${ }^{10}$ McMahon JM, Braceland FI, Moersch HJ. The psycho- somatic aspects of cardiospasm. Ann Intern Med 1951; 34: 608-30.

${ }^{11}$ Gillies M, Nicks R, Skyring A. Clinical, manometric, and pathological studies in diffuse oesophageal spasm. Br Med J 1967; 1: 527-30.

${ }^{12}$ Weiss E. Cardiospasm. Am J Dig Dis 1958; 3: 275-84. 